\title{
Article \\ Enhancement of Planar Orientation of Reactive Mesogen Molecules for Optical Retarder Film by Anisotropic Surface Plasma Treatment
}

\author{
Jiyeon Kim and Ji-Hoon Lee *(i) \\ Future Semiconductor Convergence Technology Research Center, Division of Electronics Engineering, \\ Jeonbuk National University, Jeonju 54896, Korea; elluse@jbnu.ac.kr \\ * Correspondence: jihoonlee@jbnu.ac.kr
}

Citation: Kim, J.; Lee, J.-H

Enhancement of Planar Orientation of

Reactive Mesogen Molecules for

Optical Retarder Film by Anisotropic Surface Plasma Treatment. Crystals 2021, 11, 1080. https://doi.org/ $10.3390 /$ cryst11091080

Academic Editor: Giuseppe Prestopino

Received: 29 July 2021

Accepted: 2 September 2021

Published: 6 September 2021

Publisher's Note: MDPI stays neutral with regard to jurisdictional claims in published maps and institutional affiliations.

Copyright: (c) 2021 by the authors. Licensee MDPI, Basel, Switzerland. This article is an open access article distributed under the terms and conditions of the Creative Commons Attribution (CC BY) license (https:/ / creativecommons.org/licenses/by/ $4.0 /)$.

\begin{abstract}
We proposed a method for enhancing the planar orientation of reactive mesogen (RM) molecules by means of anisotropic plasma treatment. Anisotropic surface plasma, of which energy density is dependent on the azimuthal angle, was generated by column-shaped ceramic electrodes. The anisotropic plasma was discharged on the surface of a polyvinyl alcohol (PVA) alignment layer before the rubbing process began. The contact angle of the surface was increased from $12^{\circ}$ to $83^{\circ}$ after plasma treatment, indicating a hydrophobic property of the surface. From the atomic force microscopy (AFM) measurement, it was found that the grain size of the PVA layer was reduced and that the grooved patterns were formed provided that the plasma direction was parallel to the rubbing direction of the surface. Consequently, the planar orientation was enhanced, and the in-plane retardation of the photo-polymerized RM films increased when the parallel plasma was treated on the surface.
\end{abstract}

Keywords: reactive mesogen; anisotropic surface plasma treatment; retardation; retarder film

\section{Introduction}

Optical retarder films have been widely used in the display devices [1-3]. Commercial retarder films for display devices can be classified into the stretched film type [4-6] and the reactive mesogen (RM) type [7-9]. Stretched film type retarders were developed earlier than the RM type retarders and are mainly applied to compensate for the viewing angle of liquid crystal display (LCD) $[4-6,10]$. Various kinds of polymers, such as cyclo olefin polymer (COP), polycarbonate (PC), and triacetyl cellulose (TAC), have been developed for various kinds of LCD modes. The RM type retarders have recently drawn much attention for use with organic light emitting diode (OLED) displays and flexible displays [7-9,11]. Because the thickness of RM type retarders is about one-tenth thinner than that of the stretched film type, RM type retarders are suitable for flexible display applications. In addition, RM type retarders can be fabricated by a coating process without a stretching process, which is advantageous for production costs. RM type retarders can be also used for geometric phase retarders, wherein the nanoscale periodic domains are formed with the help of the photo-alignment technique [12-14].

Meanwhile, the alignment of the RM molecules is essential, similar to the LCD fabrication process. The liquid crystal (LC) molecules are aligned by the upper and the lower substrates in the LCD, but the RM molecules in the retarder film can only be aligned by the lower substrate $[7-9,15]$. Consequently, it is difficult to fabricate a RM retarder film with a uniform orientation of the RM molecules without defects. In particular, the smectic RM molecules that are used for the coatable polarizer often represent a homeotropic orientation at the air interface [16-18]. Although the smectic RM molecules show greater order parameters than the nematic RM molecules, the homeotropic orientation at the air interface limits their application in the coatable polarizer and in the retarder film [16-18]. 
Meanwhile, there has been approaches to improve the alignment of LC molecules using plasma [19-24]. Yaroshchuk et al. applied a vacuum plasma treatment for LC alignment [19]. This idea was also applied to the alignment of RM molecules [20]. Then, it was reported that the uniform LC orientation can be obtained by the atmospheric plasma treatment $[21,22]$. In addition, it was also reported that the continuous control of the LC pretilt angle could be achieved by the combination of the plasma treatment and a rubbing treatment $[23,24]$. The effect of plasma treatment conditions such as the irradiation energy and the incident angle has been studied in the previous literature [23,24].

In this paper, we tested the surface plasma treatment, which was generated from a column-shaped ceramic electrode. The plasma could be anisotropically discharged onto the alignment layer wherein the plasma energy density is dependent on the azimuthal angle. In the previous literature [23], it was reported that a planar alignment could be induced by the oblique incidence of plasma. This result implies that some physical anisotropy can be imposed on the surface by oblique plasma treatment. In this paper, we used a columnshaped ceramic electrode for direct investigation of the effect of the in-plane plasma energy density anisotropy. The alignment of the RM molecules was investigated by varying the RM materials and the alignment layers. It was found that anisotropic surface plasma treatment can improve the planar alignment of the RM molecules, resulting in greater in-plane retardation $\mathrm{R}_{\mathrm{in}} \equiv \Delta n d$, where $\Delta n=n_{x}-n_{y}$ is the in-plane birefringence and $d$ is the thickness of the retarder. The physical reason for the retardation increase was analyzed using contact angle measurement, Fourier transform-infrared (FT-IR) absorption spectrum, and surface topology data by atomic force microscopy (AFM).

\section{Materials and Methods}

Commercially available nematic RM (HCM009, HCCH) and smectic RM (HCM026, $\mathrm{HCCH}$ ) were used in the experiment Figure 1 A quantity of $2.0 \mathrm{wt} \%$ commercial photoinitiator (Irgacure651, Ciba Chem) was mixed with RM in the toluene solvent. The concentration of the solid contents including the RM and the photoinitiator was $10 \mathrm{wt} \%$. The phase transition temperatures of the HCM009 and the HCM026 were Cry $\left(73{ }^{\circ} \mathrm{C}\right), \mathrm{N}\left(116^{\circ} \mathrm{C}\right)$, Iso and Cry $\left(72{ }^{\circ} \mathrm{C}\right), \operatorname{SmX} 1\left(78^{\circ} \mathrm{C}\right), \operatorname{SmX} 2\left(139^{\circ} \mathrm{C}\right), \mathrm{N}\left(152^{\circ} \mathrm{C}\right)$, and Iso. For the alignment of the RM molecules, a commercial polyvinyl alcohol (PVA, Aldrich) was used. The molar mass and the degree of hydration were $89,000-98,000 \mathrm{~g} / \mathrm{mol}$ and $99.0-99.8 \%$, respectively. The PVA was dissolved in distilled water at a weight ratio of $10 \mathrm{wt} \%$, spin-coated onto a glass substrate, and baked at $120^{\circ} \mathrm{C}$ for min.

(a)

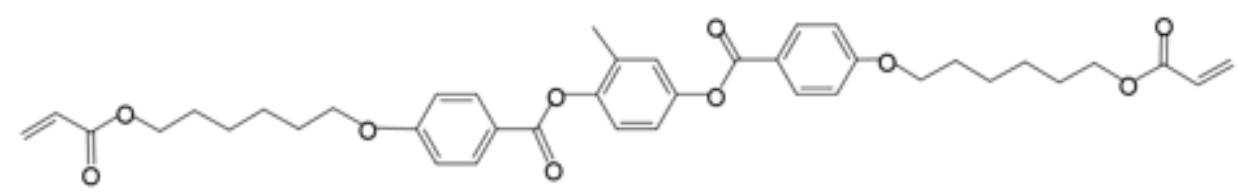

(b)

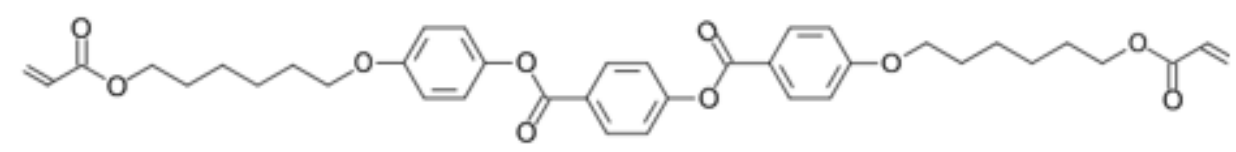

Figure 1. Chemical structure of the (a) nematic RM (HCM009) and the (b) smectic RM (HCM026) molecules used in this study.

To compare the effect of the plasma, one of the substrates was not treated with plasma and was rubbed with a cotton cloth upon baking. The other baked substrates were subjected to plasma surface treatment and then were rubbed unidirectionally with a cotton cloth. Figure 2a shows a photograph of the plasma discharging on the substrate. Atmospheric pressure plasma was applied using a commercial plasma generator (Plasma Life) [Figure 2]. 
The plasma was discharged from ceramic electrode to bottom metal stage. The length of the ceramic electrode was $300 \mathrm{~mm}$, and there were grooves with a width of $3 \mathrm{~mm}$, a separation of $3 \mathrm{~mm}$, and a depth of $3 \mathrm{~mm}$. The sample stage was open to the atmosphere, and no gas was purged during the plasma treatment. It should be noted that the plasma was discharged with an anisotropic shape elongated along the grooves of the ceramic electrodes. Thus, the plasma energy density was high along the $y$-axis direction due to the structure of the grooved ceramic electrodes. We call the elongated direction where the plasma energy density was high the plasma direction here in after. The sample was moved to the $x$-axis direction at a speed of $1.6 \mathrm{~cm} / \mathrm{s}$. The current applied to the plasma electrode was $1.05 \mathrm{~A}$, and the distance between the electrode and the stage was $5 \mathrm{~mm}$. Because the thickness of the substrate was $1 \mathrm{~mm}$, the distance from the electrode to the top of the substrate was 4 $\mathrm{mm}$. Figure $2 \mathrm{~b}$ is a schematic illustration of the plasma treatment condition. The surface of the alignment layer was treated with anisotropically discharging plasma.

(a)

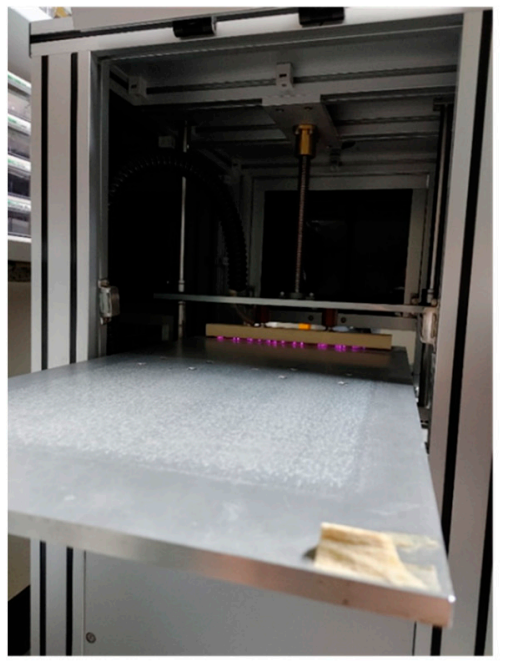

(b)
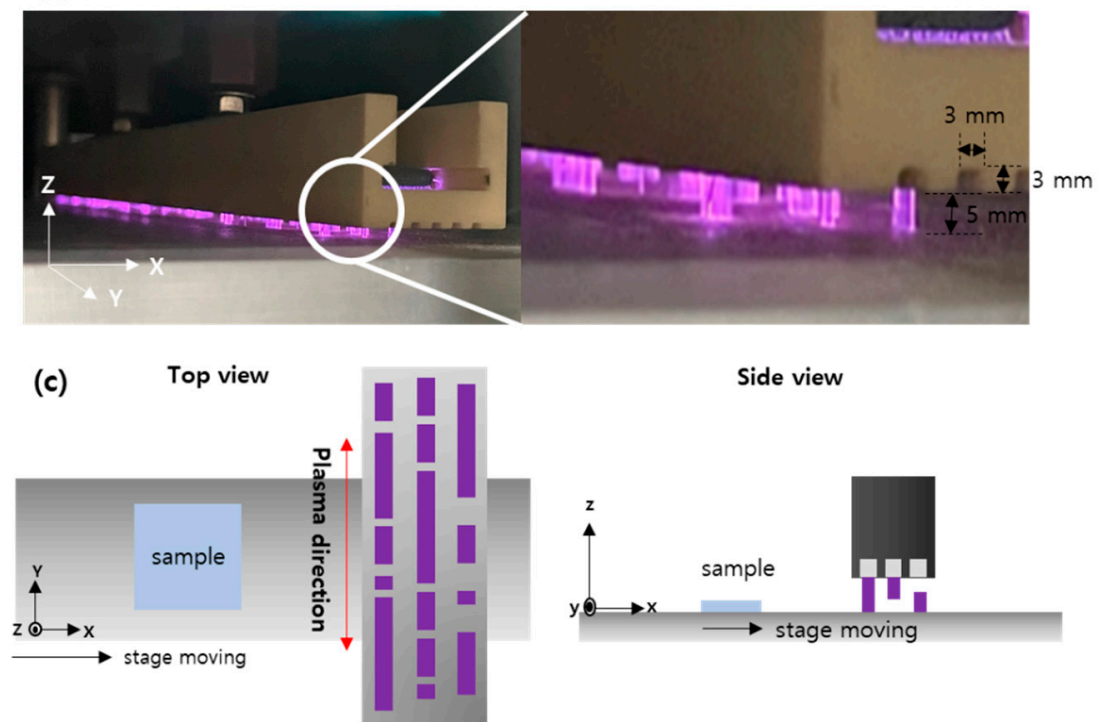

Side view

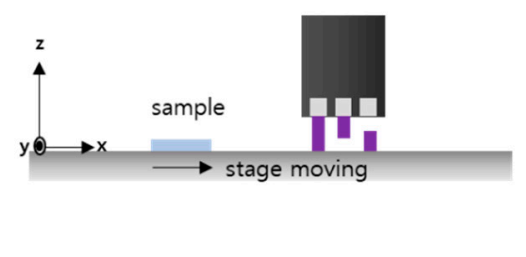

Figure 2. (a) Atmospheric plasma discharging machine used for this study. (b) Photograph of the plasma discharging on the alignment layer. It can be easily seen that the plasma is discharged with an anisotropic shape due to the column-shaped ceramic plate. (c) Schematic illustration of the experimental setup where the anisotropic plasma (AP) is discharged on the sample surface.

Thereafter, the RM molecules were diluted in chloroform at a weight ratio of $10 \mathrm{wt} \%$ and were then spin-coated on the substrates. The solvent was dried at $70{ }^{\circ} \mathrm{C}$ for $3 \mathrm{~min}$. The HCM009-coated substrate was heated to the nematic phase $\left(85^{\circ} \mathrm{C}\right)$ at a rate of $10^{\circ} \mathrm{C} / \mathrm{min}$. The temperature was maintained for $5 \mathrm{~min}$ for stabilization, and then UV light (UVITEC) was exposed to the sample for $30 \mathrm{~min}$ with the nitrogen gas purged. The wavelength of the UV light was $365 \mathrm{~nm}$, and the intensity was $4 \mathrm{~mW} / \mathrm{cm}^{2}$. The HCM026 RM film was UV polymerized at $75{ }^{\circ} \mathrm{C}$, which corresponds to the smectic phase. The HCM026 sample was pre-heated up to $152{ }^{\circ} \mathrm{C}$ and was then cooled to the UV polymerization temperature for better alignment. The HCM026 showed heterogeneous crystal textures in the first heating process and showed a homogenous orientation in the cooling process.

The measurement method of $R_{\text {in }}$ is identical to what has been reported in the previous literature $[25,26]$. A white light passed through a bandpass filter, a linear polarizer, the sample, and a polarimeter (PAX1000, Thorlab). The optic axis of the sample was placed parallel to the $x$-axis. The transmission axis of the polarizer was $45^{\circ}$ from the optic axis of the sample. $R_{\text {in }}$ was obtained by measuring the Stokes parameters of the output beam [25].

For the contact angle measurement, a commercial instrument (Sdlap-2000TEZD, Smartdrop) was used. A water droplet was dropped on the alignment layer, and the contact 
angle was checked by analyzing the captured image. For the measurement of the FT-IR absorption spectrum, a calcium fluoride $\left(\mathrm{CaF}_{2}\right)$ substrate (Edmund Optics) was used. The FT-IR absorption spectrum of the alignment layer-coated $\mathrm{CaF}_{2}$ substrate was measured before and after the plasma treatment. For investigating the surface topology of the alignment layer, atomic force microscopy (AFM) was used with a commercial AFM instrument (XE07, Parker Systems). The surface topology of the samples with various surface treatment conditions was measured.

\section{Results and Discussion}

Figure 3 shows the polarizing optical microscopy (POM) images of the nematic RMHCM009 samples located between the crossed polarizers. There were four kinds of samples that were prepared. The first sample was plasma-treated but not rubbed (Figure 3a,b). The second sample was rubbed without the plasma treatment (Figure 3c,d). The third sample was plasma-treated and then rubbed, and the plasma direction and the rubbing direction were perpendicular (Figure 3e,f). The last sample was plasma-treated and rubbed, and their directions were parallel (Figure $3 \mathrm{~g}, \mathrm{~h}$ ). The samples were rubbed at direction of $45^{\circ}$ in samples $(\mathrm{a}, \mathrm{c}, \mathrm{e}, \mathrm{g})$ and at $0^{\circ}$ in $(\mathrm{b}, \mathrm{d}, \mathrm{f}, \mathrm{h})$.

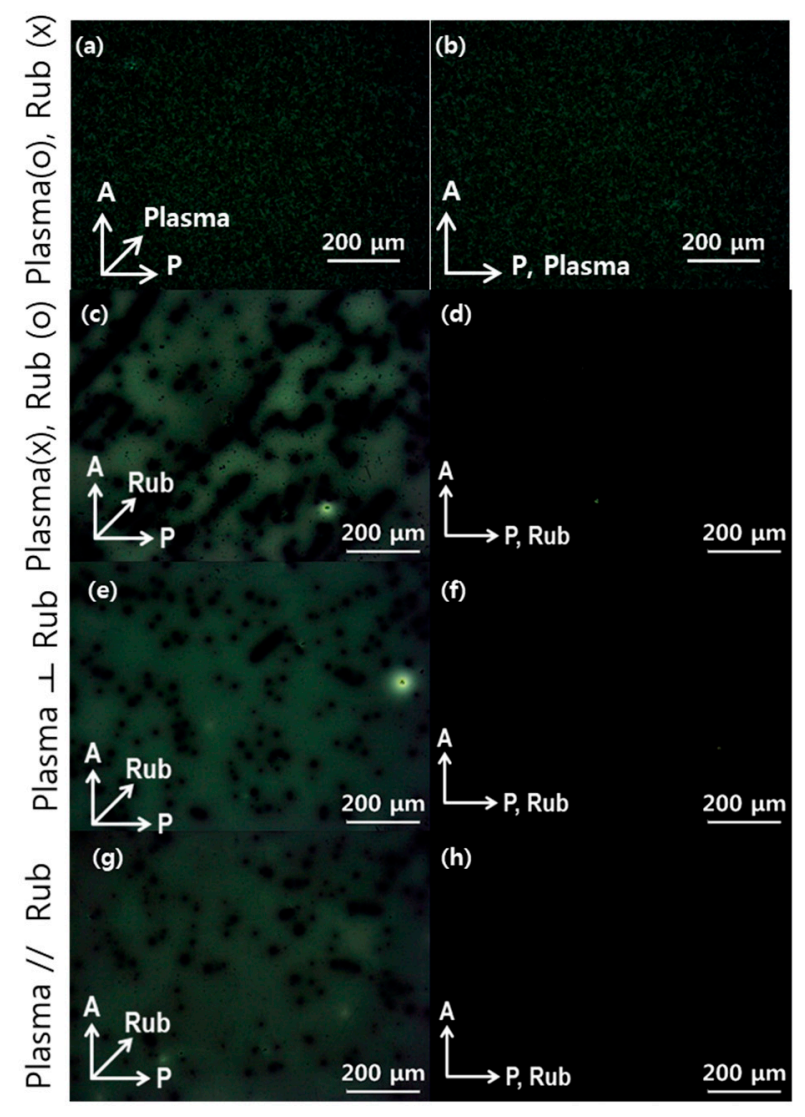

Figure 3. Polarizing optical microscopy (POM) images of the HCM009 samples located between crossed polarizers. PVA was used as an alignment layer. Plasma was treated but not rubbed in $(\mathbf{a}, \mathbf{b})$. Rubbed but plasma was not treated (c,d). The plasma direction was perpendicular to the rubbing direction in $(\mathbf{e}, \mathbf{f})$, while it was parallel to the rubbing direction in $(\mathbf{g}, \mathbf{h})$. The rubbing direction of the samples was at $45^{\circ}$ in $(\mathbf{a}, \mathbf{c}, \mathbf{e}, \mathbf{g})$ and $0^{\circ}$ in $(\mathbf{b}, \mathbf{d}, \mathbf{f}, \mathbf{h})$.

It can be observed that the sample that was plasma-treated without rubbing showed heterogeneous alignment with no extinction between the crossed polarizers (Figure 3a,b). This means that the plasma treatment could not induce an azimuthal orientation. The brightness of the sample was not increased when it was zenithally tilted between the crossed polarizers. No change of brightness was observed via the rotation of the sample at 
a tilted state. This means the sample was not in the homeotropic state but instead in the heterogenous state. On the other hand, when the sample was rubbed without the plasmatreatment, it showed a brighter texture but still showed many dark spots (Figure 3c). The sample became dark when the rubbing direction was parallel to the transmission axis of the polarizer (Figure 3d). Thus, the sample showed a planar orientation with a uniform azimuthal orientation. The area with the dark spots was certainly reduced in the samples treated with both plasma and rubbing (Figure 3e,g). In particular, the sample with the plasma direction parallel to the rubbing direction showed less dark spots than the sample with a perpendicular plasma direction. The POM observation confirmed that the planar orientation with a uniform azimuthal orientation could be induced by the cooperation of the plasma-treatment and the rubbing.

For more quantitative analysis, we measured the $R_{\text {in }}$ of the samples (Figure 4). Because the sample treated with plasma showed a homeotropic orientation without in-plane retardation, we have not plotted the data in Figure 4. Similar to the POM texture in Figure 3, the sample that was rubbed parallel to the plasma treatment direction showed a greater $R_{\text {in }}$ than the other samples. The sample rubbed without plasma treatment showed the smallest $R_{\text {in }}$ value through the visible wavelength range. We fabricated three samples to check the reproducibility in each condition, and the variance in the $R_{\text {in }}(550 \mathrm{~nm})$ was less than $2 \mathrm{~nm}$, which is smaller than the difference of $R_{\text {in }}(550 \mathrm{~nm})$ in Figure 4. Thus, the administration of the plasma treatment before the rubbing process was helpful for enhancing the planar alignment of the nematic RM molecules. Specifically, the plasma direction parallel to the rubbing direction resulted in a better planar orientation and a greater $R_{\text {in }}$. From the results in Figures 3 and 4, it can be confirmed that the plasma treatment parallel to the rubbing direction promotes a better planar orientation of the nematic RM molecules.

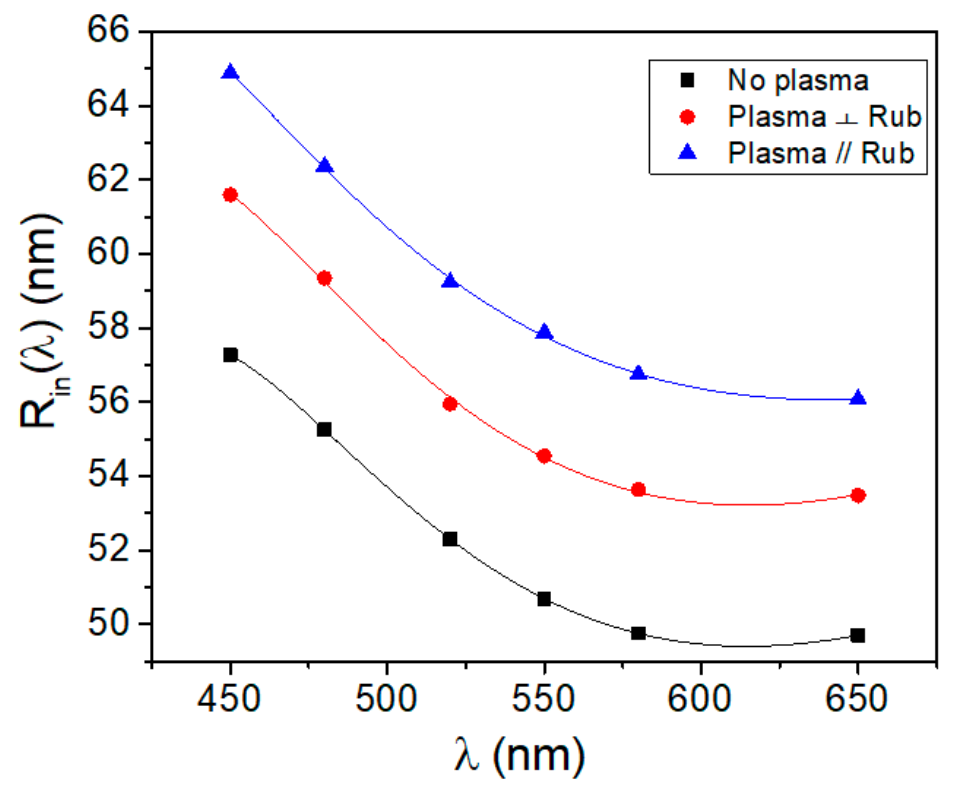

Figure 4. In-plane retardation $\mathrm{R}_{\mathrm{in}}$ of the UV-polymerized HCM009 samples with different surface treatment conditions vs. light wavelengths.

We also investigated the effect of the plasma surface treatment when a smectic RM was used. The HCM026 was used as the smectic RM material, as described in the experimental section. Figure 5 represents the POM images of the HCM026 samples located between the crossed polarizers. There were three types of samples that were prepared, similar to the nematic RM samples. The first sample was rubbed without the plasma treatment [Figure $5 \mathrm{a}, \mathrm{b}$ ]. The second sample was plasma-treated and then rubbed, and the plasma direction and the rubbing direction were perpendicular [Figure $5 c, d]$. The last sample was plasma-treated and rubbed, and their directions were parallel [Figure 5e,f]. 


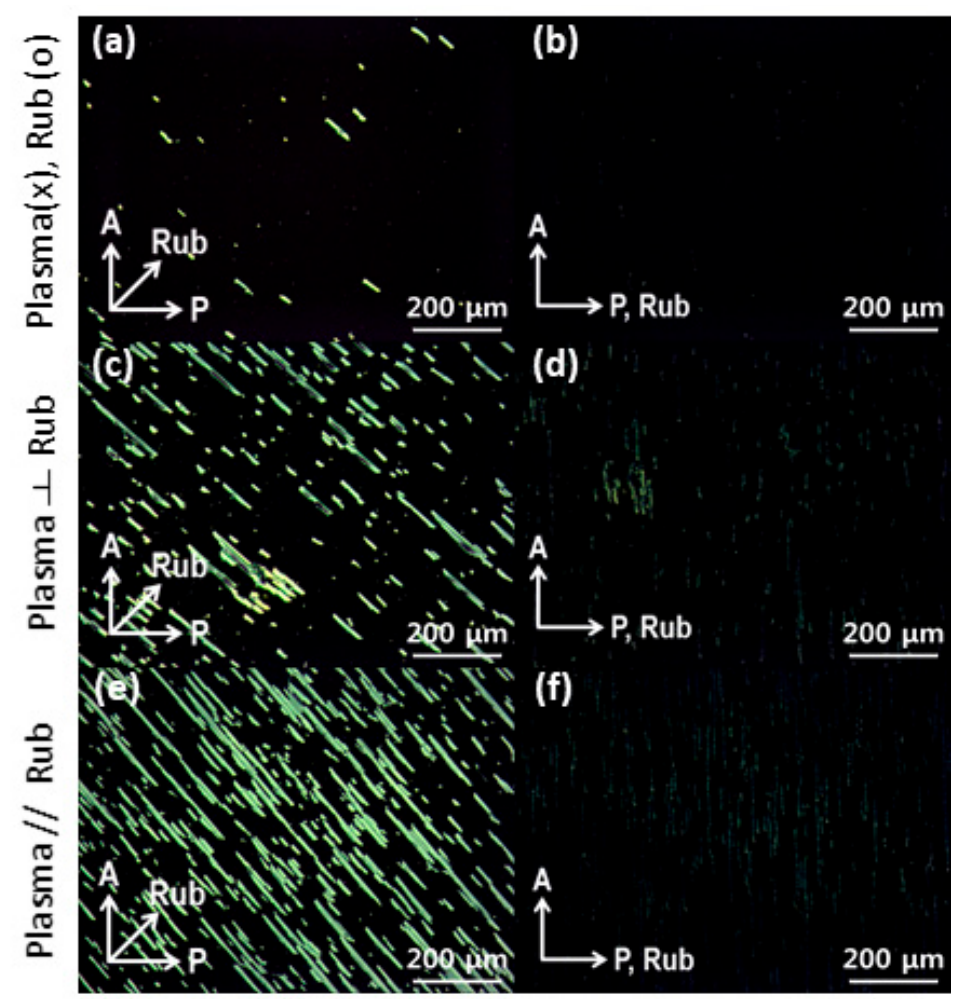

Figure 5. POM images of the HCM026 samples located between the crossed polarizers. PVA was used as an alignment layer. Plasma was not treated in $(\mathbf{a}, \mathbf{b})$. The plasma direction was perpendicular to the rubbing direction in $(\mathbf{c}, \mathbf{d})$, while it was parallel to the rubbing direction in $(\mathbf{e}, \mathbf{f})$. The rubbing directions of the samples were at $45^{\circ}$ in $(\mathbf{a}, \mathbf{c}, \mathbf{d})$ and $0^{\circ}$ in $(\mathbf{b}, \mathbf{d}, \mathbf{f})$.

The POM textures of the samples made from the smectic RM were quite different from the ones made from the nematic RM. First, the sample that was rubbed without the plasma treatment showed very little contrast when the sample was rotated between the crossed polarizers (Figure $5 a, b$ ). When the sample was zenithally tilted between the crossed polarizers, the sample became bright. Thus, most of the dark areas correspond to the homeotropic aligned RM molecules. On the other hand, the fraction of the bright domains was highly increased in the sample with the perpendicular plasma treatment (Figure 5c). It also should be noted that the fraction of the bright spots was the largest in the sample with parallel plasma treatment (Figure 5e). The bright spots became dark when the rubbing direction was parallel to the transmission axis of the polarizer (Figure $5 b, d, f$ ). Thus, the smectic RM molecules are aligned parallel to the rubbing direction.

Because the orientation of the smectic RM samples was poor, the retardation measurement was not useful for quantifying the alignment quality of the RM molecules. Instead, we analyzed the average brightness of the POM textures in Figure 5 using the Matlab program. Figure 6a corresponds to the brightness of the three types of samples when the rubbing direction of the sample was at $45^{\circ}$ and $0^{\circ}$. Figure $6 \mathrm{~b}$ represents the contrast ratio of the brightness between the bright and dark state in Figure 6a. The greater contrast ratio means greater optical anisotropy and a higher fraction of bright spots. The sample without the plasma treatment showed the smallest contrast among the samples, while the one with the parallel plasma treatment showed the greatest contrast. Therefore, it is confirmed that the parallel plasma treatment promotes the in-plane optical anisotropy of the smectic as well the nematic RM molecules. 
(a)

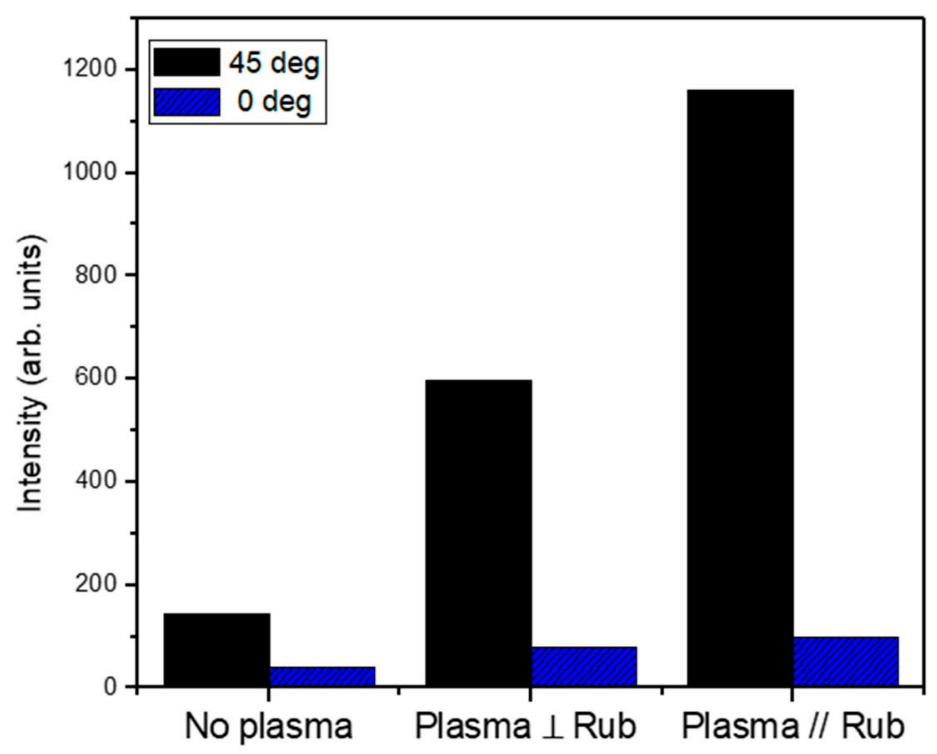

(b)

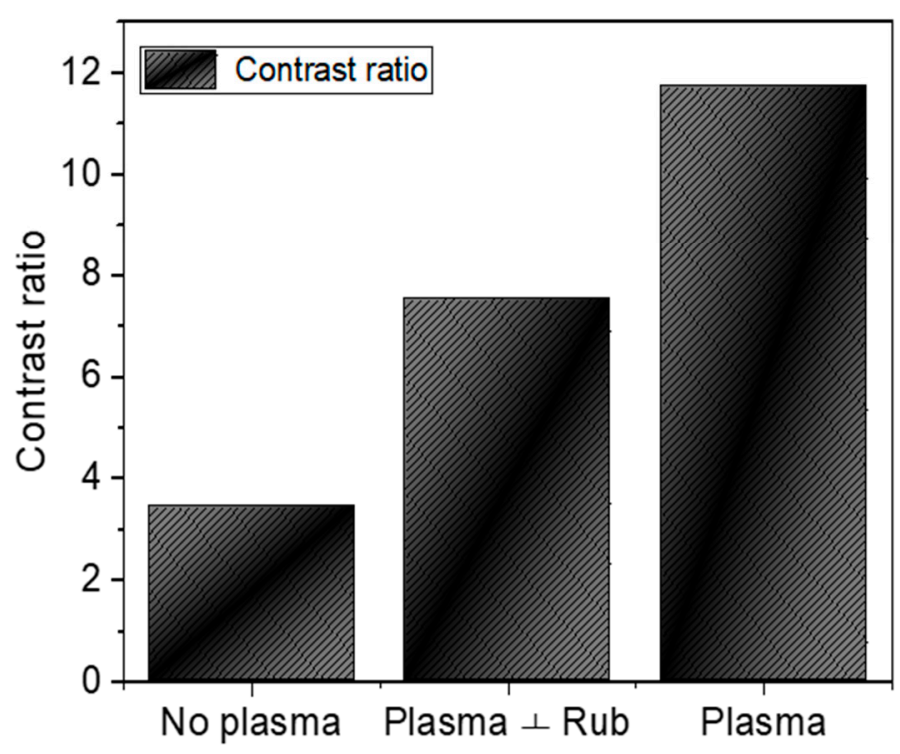

Figure 6. (a) Averaged brightness of the POM textures when the rubbing direction of the sample was at $45^{\circ}$ and $0^{\circ}$ to the transmission axis of the polarizer in Figure 5. (b) Contrast ratio between bright and dark state obtained from the data in Figure 6a.

We also varied the number of plasma treatment times before rubbing. The number of passes through the plasma discharge was varied (Figure 7). The fraction of the bright domains increased as the number of the plasma treatments increased by up to two times (Figure 7e). However, the bright spots did not increase, regardless of the number if plasma treatments (Figure 7g,i). In addition, light leakage was also observed as the frequency of the plasma treatments increased, resulting in poor contrast. 


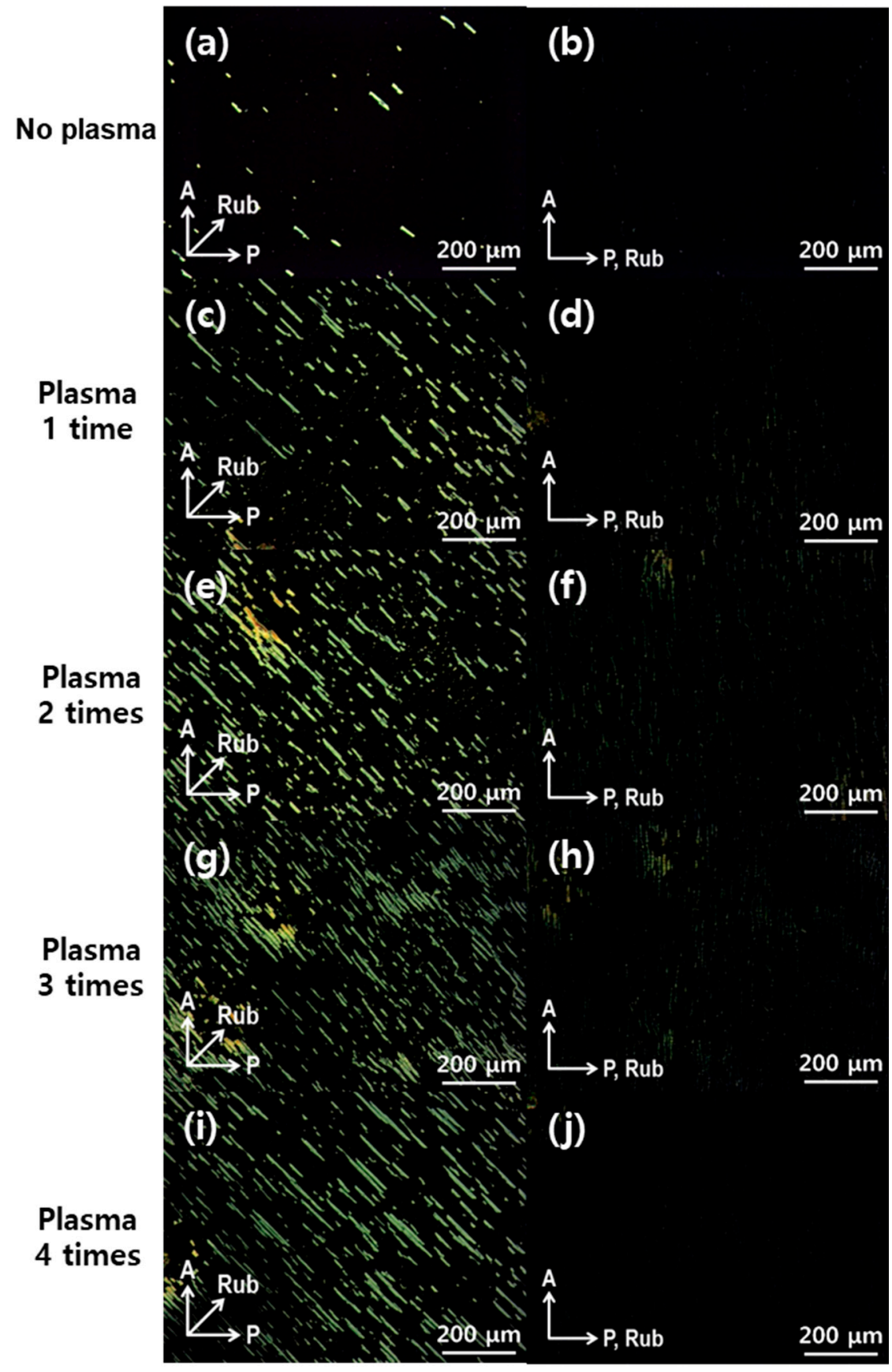

Figure 7. POM images of the HCM026 samples located between the crossed polarizers. The number of plasma treatment times was varied before rubbing. $(\mathbf{a}, \mathbf{b})$ No plasma treatment and plasma treated $(\mathbf{c}, \mathbf{d}) 1,(\mathbf{e}, \mathbf{f}) 2,(\mathbf{g}, \mathbf{h}) 3$, and $(\mathbf{i}, \mathbf{j}) 4$ times.

In order to understand the physical reason for the different alignments of the RM molecules, we investigated the changes of the surface properties using contact angle measurement (Figure 8). Figure 8 shows the contact angle property of the PVA alignment layer with different surface treatment conditions. When the plasma was not applied, the PVA layer showed a small contact angle of about $12 \sim 13^{\circ}$ (Figure $8 a, b$ ). The change of the contact angle before and after rubbing was small. On the other hand, the contact angle dramatically increased after plasma treatment (Figure $8 c, d$ ). The contact angle of the PVA-coated substrate was 85.2 and 45.2 after perpendicular and parallel plasma treatment, respectively. Because a water droplet was dropped for the measurement, the greater contact 
angle means that the surface is more hydrophobic. Thus, the PVA surface changed from being hydrophilic to being hydrophobic after plasma treatment.

(a) Plasma (X), Rub (X)

C.A $=13.5$

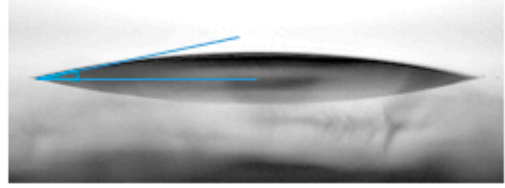

(c) Plasma $\perp$ Rub

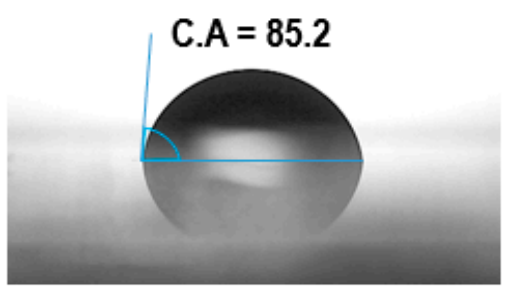

(b) Plasma (X), Rub (0)

C.A $=12.2$

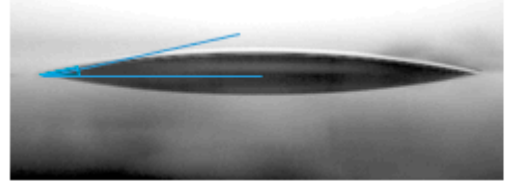

(d) Plasma // Rub

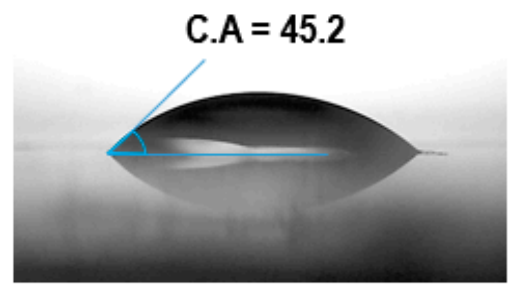

Figure 8. Captured image of the contact angle measurements on the PVA-coated surface. (a) No plasma treatment and no rubbing, (b) no plasma treatment and rubbing, (c) plasma treatement perpendicular to the rubbing, (d) plasma treatment parallel to the rubbing.

One can raise a question: if the contact angle is strongly dependent on the relative angle between the plasma and the rubbing directions, can the shape anisotropy of the water droplet be seen as it has been reported in the literature [27]? In order to respond this question, a water droplet was put on the substrate surface, and the shape of the droplet was noted after $5 \mathrm{~min}$ (Figure 9). The water droplet on the PVA surface, which was neither plasma-treated nor rubbed, showed a symmetrical shape (Figure 9a). The one on the PVA where the plasma direction was perpendicular to the rubbing direction also retained a symmetrical shape (Figure 9b). On the other hand, the droplet on the surface where the plasma and the rubbing direction was parallel showed a loosely elongated shape along the rubbing direction (Figure 9c). Thus, these observations imply that the anisotropic plasma discharge imposed some anisotropy on the PVA surface. Because the contact angle is dependent both on the chemical structure and the physical morphology, we investigated the FT-IR (Figure 10) and the AFM topography of the substrate to verify the physical reason for this (Figure 10). 
(a) Plasma (x), Rub (x)

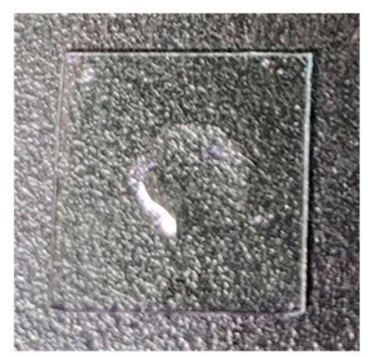

(b) Plasma (X), Rub (O)

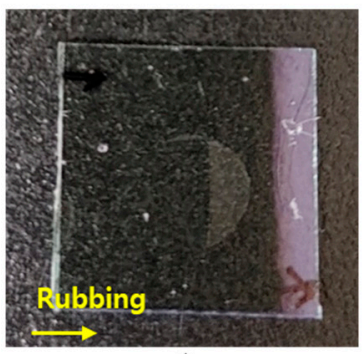

$\uparrow$ (c) Plasma $\perp$ Rub

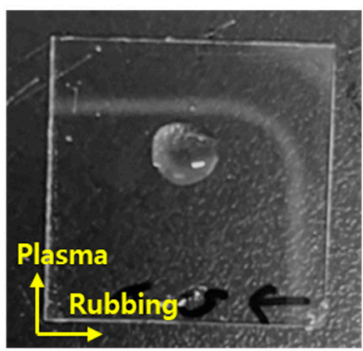

$\uparrow$ (d) Plasma // Rub

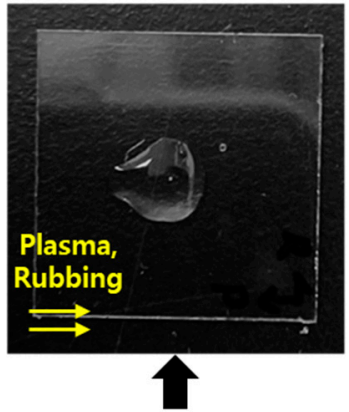

Viewing direction in contact angle measurement

Figure 9. Photograph of water droplet on the PVA-coated surface. (a) No plasma treatment and no rubbing, (b) no plasma but rubbing (c) plasma treatment perpendicular to the rubbing, (d) plasma treatment parallel to the rubbing. The photograph was taken $5 \mathrm{~min}$ after the water was dropped. Black arrows correspond to the viewing direction in the contact angle measurement.

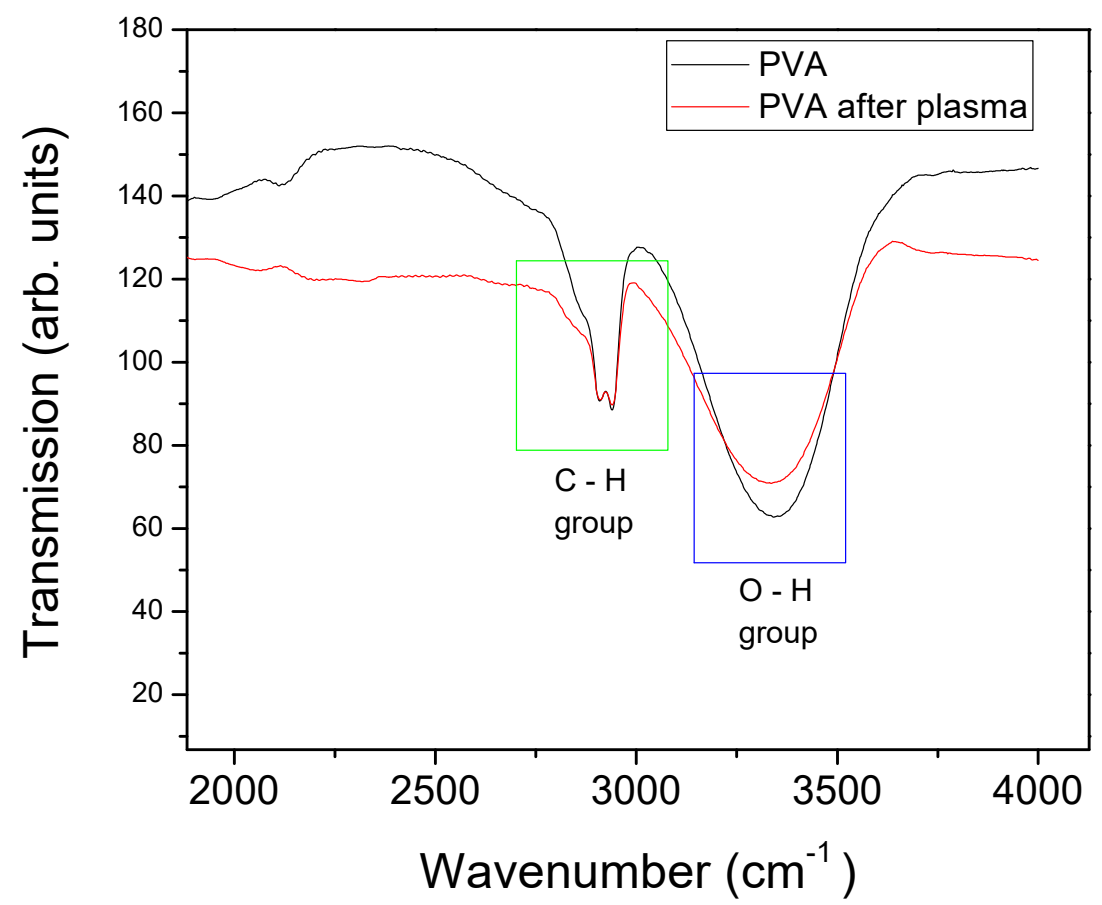

Figure 10. FT-IR transmission spectrum of the PVA polymer before and after plasma treatment.

Figure 10 represents the FT-IR transmission spectrum of the PVA layer before and after the plasma treatment. The PVA polymer that was used in this study has an OH group and is intrinsically hydrophilic. In order to check the change of the $\mathrm{OH}$ group, the spectra before and after the plasma were normalized so that the transmission at $2931 \mathrm{~cm}^{-1}(\mathrm{CH}$ group) became the same. It is observed that the transmission at $3334 \mathrm{~cm}^{-1}$ corresponding to the absorption of $\mathrm{OH}$ increased by $8 \%$ after plasma treatment, i.e., the absorption of $\mathrm{OH}$ decreased after plasma treatment. This implies that parts of the $\mathrm{OH}$ groups were decomposed by the plasma treatment. This may result in the increase of the hydrophobicity of the surface (Figure $8 c, d$ ). One may raise a question as to how to explain the change in the contact angle in Figure 8 caused by the $8 \%$ change in transmission, which seems too small to be able to do so. However, it needs to be considered that the FT-IR absorption spectrum includes absorption from the bulk and the surface of the PVA layer, while the plasma affects the surface of the PVA layer. 
Figure 11 shows the AFM topography of the PVA surface with various surface treatment conditions. When the sample was not treated by plasma, the surface morphology was smoothed by rubbing (Figure 11a-d). The grain size of the surface was reduced, and the height variance was similar after rubbing. On the other hand, when the plasma was treated perpendicular to the rubbing direction, the grain size was more reduced (Figure 11e,f). Hence, the large contact angle of this sample can be interpreted by the increase of the surface tension by the reduced size of the surface grains [28]. We should note that the the PVA layer treated with the parallel plasma (Figure $11 \mathrm{~g}, \mathrm{~h}$ ) showed quite different topography compared to the sample with the perpendicular plasma (Figure 11e,f). Although the grain size was slightly decreased comparaed to the surface without plasma treatment (Figure 11a,b), it was certainly greater than the surface treated with perpendicular plasma. Thus, the intermediate contact angle of $45.2^{\circ}$ can be interpreted with this result.

The most important observation in Figure $11 \mathrm{~g}$, $\mathrm{h}$ is the appearance of the grooved morphology along the rubbing direction. We prepared the substrate three different times, and the grooved pattern was always visible. This grooved morphlogy was also observed when the scanning direction was orthogonal to the rubbing direction, which means that the grooved pattern is not an artifact of the AFM measurement. It is commonly known that the grooved morphology on the surface can induce the unidirectional orientation of the liquid crystal molecules along the groove direction $[27,29,30]$. Thus, it is reasonable that the grooved structure obtained by the cooperation of the rubbing and the anisotropic plasma treatment parallel to the rubbing direction contributed to the planar orientation of the RM molecules. It is thought that the PVA surface is weakened by the plasma treatement along the plasma direction [31,32]. On such a soft surface, the rubbing process can result in a grooved pattern. When the rubbing direction was the same as the plasma direction, this effect became prominent, resulting in the grooved morphology. The RM molecules on such a surface can be easily aligned along the groove direction.

We further tested another kind of planar alignment layer made of polyimide (PIAX189-KU1, JNC). However, the contact angle changed before and after the plasma treatment and was less than $2^{\circ}$. In addition, the change in the $R_{\text {in }}$ was less than $4 \mathrm{~nm}$, regardless of the plasma treatment direction. On the other hand, Yaroshchuk et al. reported continuous control of the pretilt from the vertical to planar state [23]. In their report, a polymide inducing the vertical alignment of liquid crystal was used. It is thought that the side chains of the vertical alignment layer were cleaved-off by the plasma treatment, resulting in a planar alignment [33]. Thus, a more concrete conclusion can be obtained after testing various kinds of polyimide materials, which is left for the future work. Nevertheless, the proposed results can provide clues for improving the planar alignment of RM molecules for use in retarder applications. 


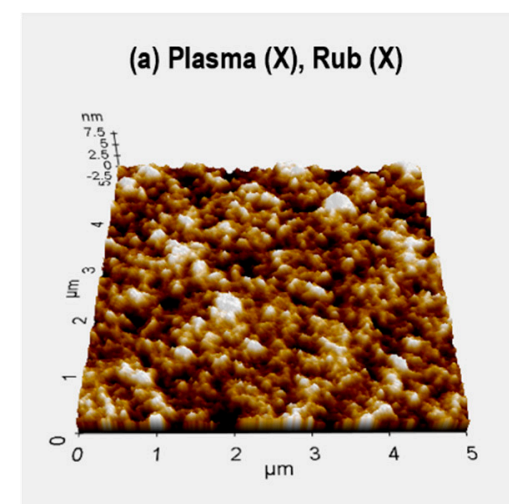

(c) Plasma (X), Rub (O)

(b) Plasma (X), Rub (X)

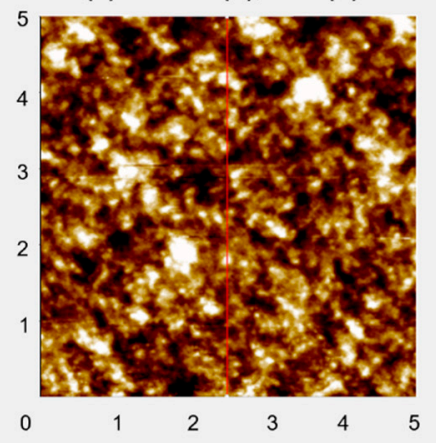

(d) Plasma (X), Rub (O)

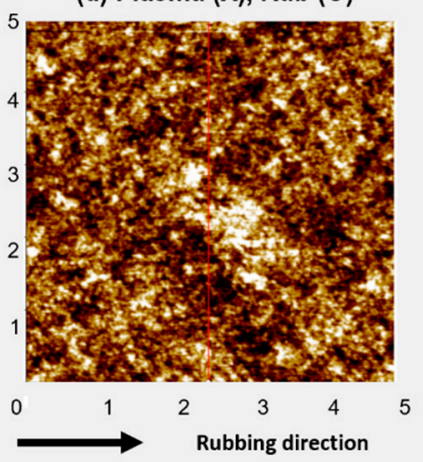

(f) Plasma $\perp$ Rub

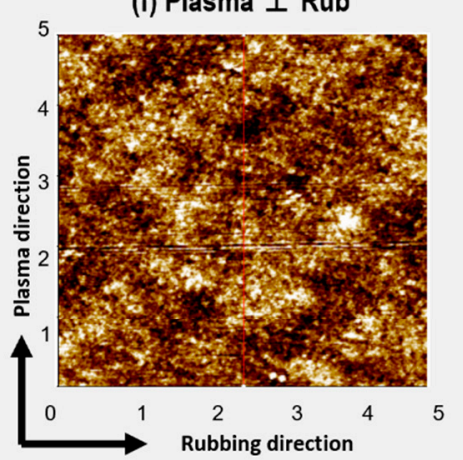

(h) Plasma // Rub

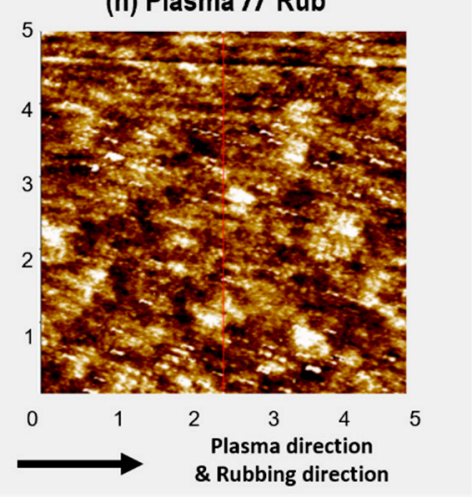

Figure 11. AFM surface topography of the PVA surface with various surface treatment conditions. $(\mathbf{a}, \mathbf{b})$ No plasma treatment and no rubbing, $(\mathbf{c}, \mathbf{d})$ no plasma treatment and rubbing, $(\mathbf{e}, \mathbf{f})$ plasma treatment perpendicular to the rubbing, $(\mathbf{g}, \mathbf{h})$ plasma treatment parallel to the rubbing. $(\mathbf{a}, \mathbf{c}, \mathbf{e}, \mathbf{g})$ are three-dimensional top-view images, and $(\mathbf{b}, \mathbf{d}, \mathbf{f}, \mathbf{h})$ are two-dimensional oblique-view images. 


\section{Conclusions}

We suggested a method that can enhance the planar alignment of nematic RM molecules by means of anisotropic plasma surface treatment. The grain size of the PVA alignment layer was reduced, and the PVA layer was softened by the plasma treatment. Grooved patterns formed on the PVA alignment layer due to the cooperation of the rubbing and the plasma parallel to the rubbing direction, resulting in a more uniform orientation of the nematic RM molecules. The proposed method can be helpful for obtaining a uniform orientation of the nematic RM molecules for use in retardation film applications.

Author Contributions: Sample fabrication, J.K.; analysis, J.K. and J.-H.L.; writing, J.-H.L. All authors have read and agreed to the published version of the manuscript.

Funding: National Research Foundation (NRF, 2019R1A2B5B01069580, 2019R1A6A1A09031717), Ministry of Trade, Industry, and Energy (20011031).

Acknowledgments: This study was supported by a grant from the National Research Foundation (NRF, 2019R1A2B5B01069580, 2019R1A6A1A09031717), Ministry of Trade, Industry, and Energy (20011031).

Conflicts of Interest: The authors declare no conflict of interest.

\section{References}

1. Yang, D.K.; Wu, S.-T. Fundamentals of Liquid Crystal Devices, 2nd ed.; John Wiley\& Sons, Ltd.: Chichester, UK, 2015; pp. 2-4; ISBN 978-1-118-75200-5.

2. Zhu, X.; Ge, Z.; Wu, S.-T. Analytical solutions for uniaxial-film-compensated wide-view liquid crystal displays. J. Disp. Technol. 2006, 2, 2-20. [CrossRef]

3. Yoon, T.-H.; Lee, G.-D.; Kim, J.C. Nontwist quarter-wave liquid-crystal cell for a high-contrast reflective display. Opt. Lett. 2000, 25, 1547-1549. [CrossRef] [PubMed]

4. Uchiyama, A.; Ono, Y.; Ikeda, Y.; Shuto, H.; Yahata, K. Copolycarbonate optical films developed using birefringence dispersion control. Polym. J. 2012, 44, 995-1008. [CrossRef]

5. Uchiyama, A.; Yatabe, T. Control of wavelength dispersion of birefringence for oriented copolycarbonate films containing positive and negative birefringent units. Jpn. J. Appl. Phys. 2003, 42, 6941. [CrossRef]

6. Yoshimi, H.; Yano, S.; Fujimura, Y. Optical films for reflective LCDs to achieve high image quality. SID Int. Symp. Digest. 2002, 33, 862-865. [CrossRef]

7. Mori, H.; Itoh, Y.; Nishiura, Y.; Nakamura, T.; Shinagawa, Y.; Shinagaya, Y. Performance of a novel optical compensation film based on negative birefringence of discotic compound for wide-viewing-angle twisted-nematic liquid-crystal displays. Jpn. J. Appl. Phys. 1997, 36, 143. [CrossRef]

8. Yun, C.-J.; Song, J.-K. Functional films using reactive mesogens for display applications. J. Inf. Disp. 2017, 18, 119-129. [CrossRef]

9. Lee, H.; Lee, J.-H. Negative dispersion of birefringence in two-dimensionally self-organized smectic liquid crystal and monomer thin film. Opt. Lett. 2014, 39, 5146-5149. [CrossRef]

10. Yeh, P.; Gu, C. Optics of Liquid Crystal Display, 2nd ed.; John Wiley\& Sons, Ltd.: Chichester, UK, 2010; pp. 242-247. ISBN 978-0-470-18176-8.

11. Parri, O.; Smith, G.; Harding, R.; Yoon, H.-J.; Gardiner, I.; Sargent, J.; Skjonnemand, K. Patterned retarder films using reactive mesogen technology. Proc. SPIE 2011, 7956, 1. [CrossRef]

12. Kim, J.; Li, Y.; Miskiewicz, M.N.; Oh, C.; Kudenov, M.W.; Escuti, M.J. Fabrication of ideal geometric-phase holograms with arbitrary wavefronts. Optica 2015, 2, 958-964. [CrossRef]

13. Oh, C.; Escuti, M.J. Achromatic diffraction from polarization gratings with high efficiency. Opt. Lett. 2008, 33, 2287-2289. [CrossRef]

14. Kim, J.; Miskiewicz, M.N.; Serati, S.; Escuti, M.J. Nonmechanical laser beam steering based on polymer polarization gratings: Design optimization and demonstration. J. Light. Tech. 2015, 33, 2068-2077. [CrossRef]

15. Son, K.-B.; Kim, M.; Park, M.-K.; Kim, H.-R. Polarization-Dependent Microlens Array Using Reactive Mesogen Aligned by Top-Down Nanogrooves for Switchable Three-Dimensional Applications. J. Opt. Soc. Kor. 2015, 19, 265. [CrossRef]

16. Kasianova, I.; Kharatyian, E.; Geivandov, A. Lyotropic liquid crystal guest-host material and anisotropic thin films for optical applications. Liq. Cryst. 2010, 37, 1439-1451. [CrossRef]

17. Hwang, J.; Yang, S.; Choi, Y.-J.; Jeong, K.-U.; Lee, J.-H. Single layer retarder with negative dispersion of birefringence and wide field-of-view. Opt. Express 2016, 24, 19934-19939. [CrossRef] [PubMed]

18. Chang, J.B.; Namgoong, J.W.; Kim, S.H.; Park, S.H.; Wang, B.H.; Kim, J.P. Effect of dye structure on orientational behavior and transition dipole moments in coatable guest-host polarizers. Dyes. Pigm. 2015, 121, 30-37. [CrossRef] 
19. Yaroshchuk, O.; Kravchuk, R.; Dobrovolskyy, A.; Qiu, L.; Lavrentovich, O.D. Planar and tilted uniform alignment of liquid crystals by plasma-treated substrates. Liq. Cryst. 2004, 31, 859-869. [CrossRef]

20. Yaroshchuk, O.V.; Parri, O.; Kravchuk, R.M.; Satayesh, S.; Reijme, M. Plasma-beam alignment of passive liquid crystals. J. Soc. Inf. Disp. 2008, 16, 905. [CrossRef]

21. Jang, E.; Song, H.; Lee, S.-D. Pretilt control of a nematic liquid crystal on polymer layers aby atmospheric plasma irradiation. Jpn. J. Appl. Phys. 2006, 45, L1238-L1240. [CrossRef]

22. Bazhenov, V.Y.; Chaplynskyi, R.Y.; Kravchuk, R.M.; Kuzmichev, A.I.; Tsiolko, V.V.; Yaroshchuk, O.V. Atmospheric Pressure Planar Radio Frequency Discharge with Isolated Electrodes: Glow Features and Application Prospects. IEEE Trans. Plas. Sci. 2017, 45, 3218. [CrossRef]

23. Yaroshchuk, O.V.; Kravchuk, R.M.; Pogulay, S.S.; Tsiolko, V.V.; Kwok, H.S. The rubbing supplemented atmospheric plasma process for tunable liquid crystal alignment. Appl. Surf. Sci. 2011, 257, 2443-2447. [CrossRef]

24. Jian, S.-J.; Kou, C.-S.; Hwang, J.; Lee, C.-D.; Lin, W.-C. Orienting layers with adjustable pretilt angles for liquid crystals deposited by a linear atmospheric pressure plasma source. Rev. Sci. Inst. 2013, 84, 063501. [CrossRef]

25. Kim, J.; Lee, J.-H. Stokes polarimetry method for measuring in-plane retardation and out-of-plane retardation of optical wave. IEEE Trans. Instrum. Meas. 2020, 69, 9805-9812. [CrossRef]

26. Jeong, J.; Choi, Y.-J.; Jeong, K.-U.; Lee, J.-H. Conversion of retardation dispersion in self-organized smectic reactive mesogen compound and its dependence on the UV polymerization temperature and the molecular orientation. Opt. Express 2018, 26, 10661-10668. [CrossRef]

27. Wang, Z.L.; Chen, E.H.; Zhao, Y.P. The effect of surface anisotropy on contact angles and the characterization of elliptical cap droplets. Sci. China Technol. Sci. 2017, 61, 309. [CrossRef]

28. Cwikel, D.; Zhao, Q.; Liu, C.; Su, X.; Marmur, A. Comparing contact angle measurements and surface tension assessments of slid surfaces. Langmuir 2010, 26, 15289-15294. [CrossRef]

29. Ishihara, S.; Wakemoto, H.; Nakazima, K.; Matsuo, Y. The effect of rubbed polymer films on the liquid crystal alignment. Liq. Cryst. 2006, 4, 669-675. [CrossRef]

30. Hah, H.; Sung, S.-J.; Han, M.; Lee, S.; Park, J.-K. Effect of the shape of imprinted alignment layer on the molecular orientation of liquid crystal. Mater. Sci. Eng. C 2007, 27, 798-801. [CrossRef]

31. Hegemann, D.; Brunner, H.; Oehr, C. Plasma treatment of polymers to generate stable, hydrophobic surfaces. Plasmas Polym. 2001, 6, 221-235. [CrossRef]

32. Premkumar, P.S. Preparation and electrical studies on pure and oxygen plasma treated polyvinyl alcohol films. J. Mater. Res. Technol. 2019, 8, 2232-2237. [CrossRef]

33. Huang, Z.; Rosenblatt, C. Large polar pretilt for the liquid crystal homologous series alkylcyanobiphenyl. Appl. Phys. Lett. 2004, 86, 011908. [CrossRef] 